\title{
Mitteilungen
}

\section{Facharztprüfung \\ Schwerpunktprüfung zur Erlangung des Schwerpunktes Forensische Kinder- und Jugendpsychiatrie und -psychotherapie zum Facharzttitel Kinder- und Jugend- psychiatrie und -psychotherapie}

Ort: Praxis Dr. med. M. Renk, Faubourg du Lac 31, 2000 Neuenburg

Datum: Donnerstag, 7. November 2019

\section{Anmeldefrist: 30. September 2019}

Weitere Informationen finden Sie auf der Website des SIWF unter www.siwf.ch $\rightarrow$ Weiterbildung $\rightarrow$ Facharzttitel und Schwerpunkte $\rightarrow$ Kinder- und Jugendpsychiatrie und -psychotherapie

\section{Preis der Schweizerischen Gesell- schaft für Urologie}

Auf Initiative von Prof. E. Wildbolz aus Bern wurde der «Preis der Schweizerischen
Gesellschaft für Urologie» zur Förderung junger Wissenschaftler/-innen errichtet.

Der «Preis der Schweizerischen Gesellschaft für Urologie» im Betrag von 10000 CHF wird jedes zweite Jahr (ungerade Jahreszahlen) verliehen

Der Preis kann an jede/-n Arzt/Ärztin oder Naturwissenschaftler/-in verliehen werden, der/die die folgenden Teilnahmebedingungen erfüllt:

a) Zur Teilnahme sind nur Schweizer/-innen oder in der Schweiz tätige Wissenschaftler/-innen unter 40 Jahren berechtigt. Handelt es sich um eine Gemeinschaftsarbeit, so muss der/die Erstautor/-in diese Bedingungen erfüllen.

b) Die eingereichte Arbeit muss ein urologisches oder ein für die Urologie wichtiges Thema behandeln.

c) Sie muss originell sein und ein hohes wissenschaftliches Niveau haben.

d) Bis spätestens 30. Juni 2019 muss die Arbeit elektronisch an das General- sekretariat der Schweizerischen Gesellschaft für Urologie eingereicht werden (info[at]swissurology.ch).

e) Die Arbeit darf zum Zeitpunkt der Preisverteilung nicht länger als vor einem Jahr publiziert worden sein.

Das Preisrichterkollegium besteht aus dem/ der Präsidenten/-in der Gesellschaft ex officio, den Chefärzten/-innen der universitären Weiterbildungskliniken A1 sowie einem praktizierenden Mitglied der Gesellschaft, das von der Geschäftsversammlung für jeweils vier Jahre gewählt wird.

Die Beurteilung der Arbeit erfolgt nach strengen wissenschaftlichen Massstäben. Der Preis soll im allgemeinen nicht geteilt werden. Liegt keine preiswürdige Arbeit vor, verbleibt die Preissumme im Kapital der Gesellschaft.

Der Entscheid des Preisrichterkollegiums ist unanfechtbar.

Die Preisverteilung erfolgt anlässlich der Jahresversammlung der Schweizerischen Gesellschaft für Urologie. 